\title{
GESTIÓN DEL PATRIMONIO ARQUEOLÓGICO EN GRAN BRETAÑA
}

\section{ARCHAEOLOGICAL RESOURCE MANAGEMENT IN GREAT BRITAIAN}

por

\author{
GILBERTO RODRÍGUEZ GONZÁLEZ
}

RESUMEN Se expone una visión global y reducida de la arqueología en el Reino Unido, y más concretamente de la gestión a cargo de los distintos protagonistas involucrados en ella. Por un lado se abordará los organismos relacionados con la administración pública (English Heritage, Royal Commission, Autoridades Locales) y por otro el sector privado (compañías privadas, arqueólogos asesores, etc.), así como otras instituciones que intentan marcar el presente y futuro de la arqueología británica (Institute of Field Archaeologists, Council for British Archaeology, las universidades).

\begin{abstract}
This paper intends to be a general outlook through British Archaology, futhermore there will be an archaeological resource management review in Britain. On the one hand, it deals with the public sector involved in caring and management the archaeological heritage (English Heritage, Royal Commission, Local Authorities), on the other hand, it will review the private sector dealing with archaeology (Units, consultants, etc.). There will be also pointed out other institutions, such as Institute of Field Archaeologists, Council for British Archaeology, which have a remarkable influence within archaeological matters.
\end{abstract}

\section{LEGISLACIÓN}

Una revisión de la arqueología de un país no quedaría completa si no se tratara el sistema legal en la que se encuadra. Ello queda aún más patente si cabe cuando tratamos de gestión del patrimonio arqueológico. Patrimonio forma parte de un concepto global que, a modo de cajón de sastre, integra ejemplos de la acción del hombre en el pasado tan dispares como grabaciones de un "cantaor" flamenco o los restos de una construcción dejados en el subsuelo por una civilización anterior a la presente. Todo ello integra lo que ha sido definido como patrimonio cultural. 
En este apartado se abordará aquella parte de la legislación que directamente afecta al patrimonio arqueológico, que es lo que aquí nos ocupa. De este modo distinguiremos varios niveles en la legislación sobre patrimonio de cualquier país.

\section{LEGISLACIÓN INTERNACIONAL}

Gran Bretaña tiene una serie de compromisos y tratados firmados en cuestiones relacionadas con el patrimonio a nivel internacional. Éste sería un primer nivel donde básicamente se trataría de la legislación europea que adopta este país, así como de las recomendaciones y pactos que emanan de un organismo supranacional como la UNESCO. Sin embargo, Gran Bretaña es uno de los pocos países que no firmaron la convención de 1970 sobre el comercio ilegal de arte y antiguiedades. No hay que olvidar las disputas entre este país y Grecia por ejemplo, debido a los famosos Lord Elgin's marbles, es decir los frisos del Partenón que los ingleses llevaron al British Museum (Ceram 1982: 46-50). También debe recordarse que este país es uno de los que más movimiento de dinero relacionado con las ventas de antigüedades tiene. Quizás sea esta razón una de las que impidió la firma de la convención antes mencionada.

Respecto a la legislación europea, Gran Bretaña puso en práctica en 1988 una directiva (85/337/CEE) que exige desarrollar un estudio de impacto medioambiental en aquellos proyectos que tienen una grave repercusión en el medio ambiente. Los estudios de impacto en el patrimonio cultural incluyen arqueología y arquitectura.

Existen dos importantes documentos sobre los procedimientos a utilizar, por un lado está Environmental Assesment o "Estudio Medioambiental", publicado en 1980 por el Ministerio de Medioambiente, y por otro los "Estudios Medioambientales y Arqueología", publicados en 1993 por el Institute of Field Archaeologists. Junto a ello existen dos institutos de estudios medioambientales que publican revistas, organizan conferencias y cobran subscripciones muy altas a sus miembros, entre los cuales figuran gran parte de los contratistas en arqueología, empresas de ingenieros y constructores. Casi todos los proyectos de construcción de carreteras requieren este tipo de estudios, por lo que el Ministerio de Transporte publicó sus propias normas en arqueología (Dapartment of Transport 1993).

Para finalizar este apartado debemos mencionar la convención europea sobre "Protección del Patrimonio Arqueológico" firmada en Malta en 1992, que en Gran Bretaña tiene más fuerza moral que efectiva pues no ha sido ratificada aún (ICAHM 1990).

\section{LEGISLACIÓN NACIONAL}

Por lo que respecta a la legislación nacional, deben tratarse varios aspectos que son la base de todo el sistema. Conviene saber que en Gran Bretaña el propietario de un terreno lo es también de todos los materiales arqueológicos encontrados en su superficie o debajo de ella. Quizás fue ésta la razón por la cual se creó una ley sobre esta materia en fecha muy temprana, ya que la primera ley redactada sobre monumentos y protección medioambiental tiene 110 años siendo una de las más antiguas de Europa.

Cuando una zona se protege porque contiene un monumento de interés cultural, el propietario de la misma no obtiene ninguna compensación económica y tampoco tiene derecho a apelar contra tal designación. Sin embargo, el propietario no tiene la obligación de permitir el acceso al monumento por parte del público, y de hecho algunas casas privadas están dentro de esta categoría y sus depósitos arqueológicos bajo la misma también están protegidos. 


\section{- Monumentos protegidos (Scheduled Monuments):}

La protección de monumentos se rige actualmente bajo el acta de 1979 (Ancient Monuments and Archaeological Areas Act 1979), el cual consolida la legislación anterior y define categorías tales como "monumento", "monumento protegido", "monumento antiguo", "monumento custodiado" y "área de importancia arqueológica". Posteriormente, en 1983, se creó la Comisión de Edificios y Monumentos Históricos de Inglaterra (Historic Buildings and Monuments Commission for England), más conocida por English Heritage, definiendo cuáles eran sus obligaciones.

El término Scheduled Monument es el concepto básico más importante de esta legislación. Constituyen esta categoría aquellos monumentos de importancia nacional que han sido seleccionados por la Secretaría de Estado y el English Heritage. Para juzgar si un monumento es de importancia nacional se evalúan ocho criterios distintos pero relacionados entre si: condición, rareza, período, vulnerabilidad, diversidad, documentación, valor de grupo y potencialidad.

La legislación actual por un lado previene cualquier actuación que dañe de algún modo a un monumento protegido, pero no evita su abandono ni garantiza su mantenimiento, por lo que dicha ley ha sido calificada de imperfecta y es considerada una forma pasiva de legislación que necesita del apoyo de acuerdos entre el gobierno y el propietario del monumento para asegurar el mantenimiento de éste.

Que un monumento esté protegido no significa que no pueda edificarse o alterarse pidiendo permiso por escrito al Secretario de Estado. Éste a su vez puede organizar una investigación pública o public enquiry para estudiar la solicitud y consultar al English Heritage. Este permiso se requiere para realizar un estudio arqueológico, demolición, destrucción, reparación, cubrición del monumento, etc. Generalmente el permiso se concede sujeto a varias condiciones relacionadas con la duración y métodos de trabajo, por ejemplo detalles sobre la distancia y profundidad de los pivotes de cimentación.

En un monumento protegido es ilegal utilizar detectores de metales, así como en un yacimiento custodiado o en un área de interés arqueológico, sin permiso previo. Dicho permiso es también requerido cuando se van a instalar nuevas tuberías y plantar en zonas agrícolas si el método es distinto al utilizado en los cinco años anteriores. Los posibles daños que un monumento pueda tener son comunicados por los inspectores de monumentos o Field Monuments Wardens, que informan regularmente al English Heritage sobre el estado y la condición de los monumentos protegidos.

\section{- Monumentos custodiados (Guardianship Monuments):}

Éste es un tipo de monumento protegido pero que está bajo el cuidado del Estado y generalmente el público lo tiene en gran estima y admiración, por ejemplo el famoso poblado de la Edad del Hierro conocido por Maiden Castle. El acceso está permitido, generalmente exigiendo el pago de una entrada.

\section{- Áreas de Importancia Arqueológica (Areas of Archaeological Importance):}

Han sido designadas cinco ciudades en Inglaterra con este calificativo: Canterbury, Chester, Exeter, Hereford y York. En estas ciudades es necesario avisar con antelación sobre cualquier tipo de obra para que las autoridades puedan realizar excavaciones si es necesario. En tal caso los promotores saben de antemano las gestiones que deben realizar antes de empezar a trabajar, también saben que las obras serán retrasadas mientras se estudia el solar pero que más tarde, acabado el estudio arqueológico, no existirá traba alguna y la licencia de obras será otorgada. 
Argumentos a favor y en contra pueden encontrase sobre este concepto. Los argumentos a favor básicamente indican que la arqueología en esas cinco ciudades ha visto aumentar su consideración. Otra ventaja supone que las propuestas de obra pasan una especie de escrutinio arqueológico y otras parcelas que no caen exclusivamente bajo el control del planeamiento. El último punto a favor cae en el campo de lo práctico, ya que existe la posibilidad de entrar en los yacimientos urbanos con propósitos de investigación hasta por 18 semanas. Estos poderes se extienden para el estudio arqueológico de edificios y de depósitos urbanos.

Los puntos en contra argumentados pueden resumirse en que, se considera que suponen un aumento de burocracia. También todo el proceso administrativo requiere la transferencia de recursos económicos desde el gobierno central, cosa que nos se produce. Tampoco se ha presupuestado dinero cuando la autoridad competente en la conservación del patrimonio decide realizar la excavación.

\section{LEY DEL SUELO Y NORMAS URBANÍSTICAS}

La mayoría de los yacimientos arqueológicos, quizás un 98\% del número total, unos 600.000 aproximadamente, están más protegidos por la Ley del Suelo que por la del Patrimonio. Los cambios más recientes acontecidos en la Ley del Suelo culminan en una norma de orientación urbanística conocida como PPG16, Planning Policy Guidance 16, acerca de "arqueología y urbanismo" que se publicó a finales de 1990.

En este documento se reafirma la importancia de la arqueología y de la protección del patrimonio arqueológico, teniendo la responsabilidad más directa de su control las autoridades locales, quienes deben informar a todo promotor. Se contempla además por vez primera una presunción en favor a la preservación física, el concepto del entorno del monumento, el de evaluación para asesorar la importancia de los restos, y la recomendación de acuerdos para encontrar estrategias correctoras y de mitigación de los efectos negativos sobre la arqueología de un lugar. Pero, por encima de todo, se afirma en este documento que son los constructores, y no el gobierno central o local, quienes tienen que facilitar los resultados de la investigación arqueológica, y por tanto financiarla.

Estas directrices constituyen el paso más importante dado en la historia de la arqueología británica. Establecen claramente el traslado de la inversión económica del gobierno al sector privado, provocando así mismo un cambio en la mentalidad del arqueólogo. Ahora son muy populares los cursos en finanzas, dirección de proyectos y legislación, a la vez que han surgido nuevos sectores de empleo que no existían antes de 1990.

\section{LEGISLACIONES LOCALES}

A nivel de región y comarca, los planes locales tienen un número limitado de normas y directrices. Por ejemplo, las casas alrededor del Parlamento de Westminster en Londres tienen un alto potencial arqueológico, pero los constructores interesados saben por el plan urbanístico local que si solicitan permiso para trabajar en esta zona pueden encontrar trabas con la arqueología.

Quizás uno de los documentos más famosos y conocidos fue el publicado por el City Council (ayuntamiento) de la ciudad de York en colaboración con la empresa Ove Arup. Se apuntó como propósitos del estudio por una parte actualizar el conocimiento acerca de la arqueología de la ciudad de York, así como proporcionar unas reglas de juego para conseguir que el derecho al desarrollo urbano tampoco se viera afectado, buscando un equilibrio entre desarrollo y conservación (Ove Arup 1991).

Como fruto de este estudio se publicarán un año más tarde los principios para la conservación del patrimonio arqueológico de la ciudad de York (York City Council 1992). En dicho documento se señalaban 
los objetivos y propósitos, los cuales básicamente eran promover el desarrollo de la ciudad y conservar y gestionar el patrimonio arqueológico. Se daba a conocimiento público las características del patrimonio arqueológico de la ciudad, así como se dejaban sentadas las bases de la futura agenda de investigación. Continuaba dicho documento poniendo patente cuáles eran la bases legales que dirigían las acciones a desarrollar sobre dicho patrimonio.

Los protagonistas de este proceso también eran puestos cada uno en su sitio, intentando con ello clarificar de este modo los cometidos de ayuntamiento, universidad, empresas arqueológicas y English Heritage. Se reglamentaba todo tipo de procedimiento administrativo ligado a la arqueología y su estudio a través de los yacimientos urbanos.

\section{CÓDIGO PROFESIONAL}

Existen dos códigos principales para el mundo de la arqueología. El primero es el que se mantiene con los extractores de mineral y el segundo es el redactado entre arqueólogos y constructores británicos. En ellos se definen los procedimientos aceptables en la investigación arqueológica, por ejemplo cómo tratar con la prensa.

\section{PROTAGONISTAS}

Dentro del cuadro de protagonistas que intervienen dentro del mundo de la arqueología en Gran Bretaña se pueden hacer dos grandes grupos: la administración pública y el sector privado.

En el sector público:

\section{- English Heritage:}

Este organismo fue creado en 1983 como organización estatal encargada de la preservación y presentación del patrimonio. Estas funciones se desarrollan desde el gobierno para potenciar y asegurar la financiación de las mismas. Se han publicado los planes para los próximos diez años; también se revisó la labor de los últimos diez años en educación, excavaciones arqueológicas y publicaciones (English Heritage 1991a).

Todos sus proyectos se desarrollan según los pasos y normas establecidas en su manual sobre la dirección de proyectos arqueológicos, bajo el que compañías privadas y otros grupos pueden pedir financiación para proyectos arqueológicos (English Heritage 1991b). En 1992 se concedieron mil quinientos millones de pesetas en becas de este tipo, cuyos resultados se publican en una revista anual conocida como Archaeological Review.

English Heritage tiene un papel muy activo el la dirección de la arqueología del país, figurando entre sus principales áreas de interés la protección de monumentos, la arqueología en zonas urbanas y los paisajes culturales. Fruto de la acción de este organismo es el Programa de Protección de Monumentos o Monuments Protection Programme, en el que se identifican y definen todas las posibles clases de monumentos existentes y se protege una selección representativa, desde cuevas paleolíticas a defensas de la Segunda Guerra Mundial. Se espera que el número de yacimientos protegidos pase de trece mil a sesenta mil a finales de siglo (Darvill, Saunders \& Startin 1987).

Los yacimientos más grandes y complejos son los urbanos. Dentro de este tipo se desarrollaron proyectos pioneros en tres de las principales áreas urbanas del país para intentar encontrar un modelo válido en el 
análisis y organización de los depósitos urbanos (Ove Arup 1991, para York; Darvill \& Gerrard 1994, para Cirencester; Lowther et al. 1993, para Durham).

Para Escocia existe un organismo de similares características conocido como Historic Scotland, así como el $C a d w$ para Gales. Ambos son fruto del hecho diferencial que suponen Escocia y Gales dentro de Gran Bretaña. Para Irlanda del Norte es el Ministerio de Medio Ambiente el que desarrolla las funciones del English Heritage en Inglaterra.

\section{- Royal Commission:}

Esta institución está encargada de recoger los datos y la información que integran el Archivo Nacional de Monumentos o National Monuments Record, el inventario de monumentos y edificios antiguos de Inglaterra, entre los que se incluyen los yacimientos marinos. La biblioteca Nacional de Fotografías Aéreas está bajo cargo de esta institución.

\section{- Autoridades locales:}

Desde 1970 los arqueólogos territoriales o County Archaeological Officers han sido los encargados de asesorar sobre cuestiones de arqueología en todas las regiones del país. Generalmente trabajan en los departamentos de urbanismo, casi siempre en puestos de trabajo financiados por English Heritage. Sus principales funciones son el mantener y actualizar la lista de yacimientos y monumentos existentes en su región, además de examinar el potencial arqueológico en las solicitudes de licencias de obras y puntualizar las especificaciones que los arqueólogos deben cumplir en los diversos proyectos que se le presentan.

En el sector privado:

\section{- Compañías privadas:}

La arqueología se convirtió en un negocio rentable gracias a los cambio en la legislación. La mayoría de los arqueólogos que trabajan actualmente en Gran Bretaña lo hacen para compañías comerciales que trabajan en cualquier región del país.

Las mayores compañías tienen una plantilla fija de alrededor de cuarenta personas y unos doscientos millones de pesetas de ingresos anuales, éstos procedentes de evaluaciones de tipo arqueológico y medioambiental. Para sobrevivir en un mundo comercial tan altamente competitivo estas compañías necesitan desarrollar unos sistemas de dirección, presupuestos y marketing muy sofisticados.

Estas compañías no reciben ayuda oficial y son independientes, sus clientes contactan con ellas por medio del Instituto de Arqueólogos de Campo o Institute of Field Archaeologists, donde se mantienen un registro de las compañías arqueológicas existentes en cada región del país.

\section{- Arqueólogos asesores:}

Normalmente sólo aconsejan sobre problemas arqueológicos y muy raramente desarrollan trabajo de campo. A la hora de la verdad estos profesionales son los pilares del "ciclo de administración arqueológica". Son contratados por los promotores para negociar con el arqueólogo territorial, y acordar con él el tipo 
de investigación arqueológica apropiada al solar de su cliente, escriben los requerimientos necesarios para desarrollar dicho trabajo y lo ofrecen a varias compañías, controlan el trabajo de éstas y también la producción del informe final.

El asesor, contando con la aprobación del cliente, selecciona la empresa y su éxito dependerá de si es capaz de convencer a las autoridades, al arqueólogo territorial, que el estudio arqueológico que él ha diseñado ha sido el adecuado (Darvill \& Atkins 1991).

Otros grupos pueden ser individualizados, poniéndolos de forma separada de los dos grupos comentados anteriormente. Así tenemos a las universidades, el Instituto de Arqueólogos de Campo y el Consejo de Arqueología Británica :

\section{- Instituto de Arqueólogos de Campo (Institute of Field Archaeologists):}

La creación y el éxito de esta organización es una muestra patente de que la arqueología forma parte del mundo de profesionales. El Instituto cuenta actualmente con mil quinientos miembros recibiendo al año unas ciento cincuenta solicitudes, y ha establecido standards de trabajo, código de conducta, programas de formación ${ }^{r y}$ el marco disciplinario de la profesión (IFA 1988).

Existen diferentes categorías de miembros, siendo el nivel más alto el llamado MIFA, es decir, el nivel al que se accede tras la convalidación ante un comité en al menos dos áreas de competencia (bien en estudio de material, en excavación, etc.). La calidad de miembro es la más clara señal para otros profesionales, tales como arquitectos e ingenieros, de un cierto nivel dentro de la profesión. El instituto publica una revista, The Field Archaeologist, y estudios técnicos de diversos tipos como fotografía aérea, prospección geofísica, legislación, etc. Esta institución también celebra anualmente una conferencia nacional, la más importante en arqueología, a la que acuden regularmente unos quinientos profesionales.

\section{- Consejo de Arqueología Británica (Council for British Archaeology):}

Esta institución comprende todo tipo de cuerpos nacionales y locales, y se encarga de contestar preguntas del público, desarrollar aspectos educativos, plantear cuestiones al Parlamento y publicar estudios, especialmente en el campo de la metodología. Hay una revista bimensual, pero su publicación más importante es la British Archaeological Bibliography, que lista todos los artículos y libros que se publican cada año, agrupados según materias.

\section{- Universidades:}

Durante los sesenta, se produjo en el país una gran demanda de arqueólogos de campo debido a la construcción de carreteras y el desarrollo de ciudades. Sin embargo, las universidades fueron incapaces de responder a temporadas largas de trabajo y a los contratos cortos exigidos por los constructores.

El desarrollo de proyectos de investigación ha sido la principal ocupación del personal docente en las universidades, pero incluso en muchas ocasiones la escala de estos trabajos es superada en cantidad, y a veces en calidad, por los muy experimentados equipos que trabajan para las compañías privadas. 
Entre los datos suministrados por el English Heritage para 1991-92 se observó que de los mil millones de pesetas repartidos en becas o ayudas sólo el $3 \%$ fue a parar a las universidades. Sin embargo, esta cantidad aumentará cuando las universidades vean sus presupuestos recortados.

\section{CICLO DE ADMINISTRACIÓN ARQUEOLÓGICA}

Debido al énfasis que la Ley del Suelo ha tenido en la protección del patrimonio arqueológico, fue necesario adecuar la investigación arqueológica al ciclo de la construcción. De este modo se ha desarrollado un modelo conocido como el "ciclo de administración arqueológica", en el que la investigación arqueológica se organiza a través de una serie de fases sucesivas (Darvill \& Gerrard 1992). El paso de una a otra depende de los resultados obtenidos en las anteriores:

\section{(1) Valoración Arqueológica (Archaeological Appraisal):}

Su objeto es la consulta de la información disponible de un determinado solar o terreno de forma breve y concisa, por ejemplo a través de informes de excavaciones anteriores y fotografías aéreas, para determinar la presencia o ausencia de restos. Este estudio se realiza generalmente antes de que el promotor haya comprado el solar, por lo que la confidencialidad sobre el proyecto es un factor decisivo. El coste de este tipo de estudio ronda alrededor de las cincuenta mil pesetas.

\section{(2) Examen Arqueológico (Archaeological Assesment):}

Es un detallado estudio de todas las fuentes primarias y secundarias disponibles. Normalmente incluye el estudio de mapas y documentos recogidos en archivos regionales, pero también la verificación y análisis de la información arqueológica existente, transcripción de fotografías aéreas, y algunas veces prospección directa en el campo.

Todos los datos recogidos se presentan en un informe agrupados por períodos cronológicos, valorando la importancia de las fuentes consultadas. Este tipo de estudio es muy valioso si se desarrolla al comienzo del proyecto, porque permite suponer el potencial de un solar. El coste oscila entre doscientas mil pesetas y un millón de pesetas, dependiendo del tamaño y naturaleza del proyecto.

\section{- Evaluación de campo (Field Evaluation):}

Si en la fase anterior se ha detectado la existencia de depósitos arqueológicos, entonces es necesario determinar la importancia de éstos, su extensión, condición y profundidad. Para ello se utilizan distintas tácticas, por ejemplo estudio topográfico, geográfico, geofísico, limpieza de fosos y excavación entre el $2-5 \%$ de la superficie total del solar.

El tipo de trabajo a desarrollar, por ejemplo dónde colocar las catas, sus dimensiones, etc., se acuerda con el arqueólogo territorial (county archaeologist) a la vista de los resultados del "examen arqueológico". Por ejemplo se adoptarán distintas estrategias según la naturaleza de los yacimientos detectados, o las características de la tierra y geología, que por ejemplo pueden determinar el empleo de la prospección geofísica.

Dependiendo del tipo de trabajo técnico requerido, se contratarán los servicios de distintos especialistas, todos ellos coordinados por un arqueólogo asesor. Cuando los resultados obtenidos son negativos, el estudio 
arqueológico generalmente termina en esta fase y se concede licencia de obras. Los costes de este tipo de trabajo pueden resultar altos, pues el precio de la excavación urbana ronda las sesenta mil pesetas por metro cúbico excavado, incluyendo presupuesto para conservación. La prospección geofísica cuesta alrededor de ciento cuarenta mil pesetas la hectárea.

\section{- Formulación de estrategias (Strategy Formulation):}

De acuerdo con los resultados obtenidos en las tres fases anteriores, se diseñará la estrategia más apropiada a seguir ante los resultados arqueológicos obtenidos. En términos generales se pueden distinguir las siguientes estrategias de mitigación actualmente :

(1) Construcción de edificios siguiendo los métodos normales, cuando no se ha reconocido ningún potencial arqueológico en el solar.

(2) Construcción de edificios utilizando pivotes en su cimentación, taladrando agujeros en una cuadrícula de seis por seis metros, método que permite preservar el $95 \%$ de los depósitos arqueológicos. El suelo de la planta baja es fijo, por lo que una posterior reutilización del edificio mantendrá la misma cimentación.

(3) Construcción de edificios utilizando pivotes en su cimentación en una cuadrícula de seis por seis metros. El suelo de la planta baja es móvil, por lo que el solar se puede excavar en el futuro, por ejemplo si cambia el uso del edificio y se necesita construir sótanos o si se cambian los intereses académicos y ahora es necesario extraer la información del subsuelo.

(4) Construcción de edificios utilizando pivotes en una cuadrícula similar a las anteriores, pero en una secuencia que permitiría a los arqueólogos excavar a la vez que se produce la construcción del edificio.

(5) Excavación del solar antes de la construcción del edificio, permitiendo que éste pueda ser construido después sin ningún reparo.

(6) Excavación sólo parcial, dejando una parte del solar construida sobre pivotes.

(7) Combinación de cualquiera de los métodos anteriores. Cuando son proyectos de cierta envergadura, generalmente se produce una combinación de varios de estos métodos.

El coste de este informe suele oscilar entre cuatrocientas y seiscientas mil pesetas.

\section{- Ejecución de la Estrategia (Strategy Implementation):}

En esta fase se pone en marcha las recomendaciones definidas en la cuarta fase. Se puede excavar antes de que comiencen las obras de construcción, aunque también se puede vigilar, watching briefs, mientras se está trabajando en las obras, y las tareas de conservación y preparación del archivo del yacimiento pueden completarse cuando las obras han terminado. Los costes de almacenamiento del material arqueológico y su conservación corren a cargo del promotor. El coste de esta fase es difícil de calcular. En una zona urbana no es raro que oscile entre un millón y medio y cincuenta millones de pesetas, aunque no se puede olvidar que el paro de las obras o su retraso puede suponer mucho más dinero.

\section{CONCLUSIONES}

- La estructura de la arqueología británica ha cambiado de forma importante en la última década. English Heritage, uno de los principales financiadores de los estudios arqueológicos, ha reducido su presupuesto y son ahora los promotores los que suministran esa financiación. 
- Existe un nuevo profesionalismo, una nueva clase de arqueólogo bien preparado sobre la organización de proyectos, control legislativo y financiación.

- La redefinición de la profesión supone también una crisis de identidad, y ahora los arqueólogos están haciendo trabajos que nunca habían querido hacer, añorando los días de la tradicional excavación de verano ahora frenada por la legislación actual.

- La moda de excavar como método de recopilar datos está llegando a su fin, y está siendo sustituido por nuevas estrategias que salvaguardan las fuentes disponibles para generaciones futuras, examinando con los mejores métodos al alcance sólo aquellas que están amenazadas.

\section{BIBLIOGRAFÍA}

CERAM C.W. (1982): El Mundo de la Arqueología. Barcelona, Ediciones Destino.

DARVILL T., SAUNDERS A. \& STARTIN B. (1987): "A question of National Importance: Approaches to the Evaluation of Ancient Monuments for the monument protection Programme in England", Antiquity 61: 393-408.

DARVILL T. \& ATKINS M. (1991): Regulating Archaeological Work by Contract. Birmingham, IFA.

DARVILL T. \& GERRARD C. (1992): "Evaluating Archaeological Sites: The Cotswold Archaeological Trust Approach", en Darvill T. \& Holbrook N. (Eds) Cotswold Archaeological Trust Annual Review 2:, 10-14. Cirencester, CAT.

(1994): Cirencester: Town and Landscape. Cirencester, CAT.

DoE (Department of Environment) (1989): Environmental Assesment: A guide to the Procedures. London: HMSO.

(1990): Plannig Policy Guidance Note 16: Archaeology and Planning. (PPG16) London: HMSO.

ENGLISH HERITAGE (1991a): Exploring our Past: Strategies for the Archaeology of England. London: HBMC.

(1991b): The Management of Archaeological Projects. London: HBMC. $2^{\mathrm{a}}$ edición.

GERRARD C. (1993): "Arqueología en Gran Bretaña", en $1^{a}$ Reunión Internacional sobre el Patrimonio Arqueológico. Modelos de Gestión. Valencia.

ICAHM (1990): (International Committee on Archaeological Heritage Management) Charter for the Protection and Management of the Archaeological Heritage. Paris, ICOMOS.

IFA (1988): (Institute of Field Archaeologist) By-Laws of the Institute of Field Archaeologists: Code of Conduct. Birmingham, IFA.

LOWTHER P. et al. (1993): “The City of Durham: An Archaeological Survey”, en Durham Archaeological Durham 9: 27-119.

OVE ARUP (1991): York Development and Archaeological Study. Manchester.

YCC (1992): (York City Council) Conservation Policies for York: Archaeology. York, YCC. 